\title{
La importancia de informar ante la ansiedad y la resiliencia de pacientes que van a ser sometidos a una colonoscopia \\ The importance of reporting to the anxiety and the resilience of patients will undergo a colonoscopy
}

\author{
Juana María Olmo-Conesaa , Magdalena Gómez-Díaz \\ a Unidad de Digestivo, Servicio Murciano de Salud, España \\ b Facultad de Enfermería, Universidad Católica de Murcia, España
}

\section{Resumen}

Introducción: La colonoscopia es una técnica beneficiosa pero no deja de ser un procedimiento incómodo, donde el dolor y el miedo a lo desconocido han hecho que este procedimiento genere cierto grado de ansiedad en el paciente. Objetivos: Se trata de evaluar el nivel de ansiedad y resiliencia en los pacientes que van a ser sometidos a colonoscopia, y comprobar si el conocimiento y la información recibida influye en dichas variables. Metodología: Se trata de un estudio observacional, descriptivo, transversal y prospectivo, realizado en una muestra de 100 pacientes, 50 sometidos a la prueba con sedación consciente y 50 con sedación profunda, que acudieron a la realización de una colonoscopia. Los instrumentos de evaluación utilizados fueron el State-Trait Anxiety Inventory (STAI) y el Connor-Davidson Resilience Scale (CD-RISC-10). Resultados: El 52\% de los pacientes obtuvieron una ansiedad-estado elevada. Este nivel de ansiedad se relacionó de manera estadísticamente significativa con haber recibido información o no ( $p=.00)$ y con el nivel de la misma, suficiente $o$ insuficiente $(p=.00)$. Y los pacientes más resilientes resultaron ser aquellos que sí conocían la prueba o recibieron información sobre la misma. Conclusión: Podemos observar que a más información menos ansiedad y mayor nivel de resiliencia frente a una colonoscopia.

Palabras clave: ansiedad; resiliencia; colonoscopia; enfermería; información; sedación consciente; sedación profunda.

\begin{abstract}
Introduction: Colonoscopy is a highly demanded technique due to its benefits, but it is nevertheless an uncomfortable procedure where pain and fear of the unknown can create a certain degree of anxiety. Objectives: The main objective of this study is to evaluate the level of anxiety and resilience in patients going to undergo colonoscopy, and check if the received information and knowledge affects these variables. Methodology: This is an observational, descriptive, transversal and prospective study. The study sample included 100 patients, 50 tested with conscious sedation and 50 with deep sedation, who underwent a colonoscopy. The assessment tools used were: State-Trait Anxiety Inventory (STAI) y Connor-Davidson Resilience Scale (CD-RISC-10). Results: $52 \%$ of the sample experienced a high degree of anxiety. This level of anxiety related statistically significantly with receiving information or not $(p=.00)$ and with the same level, sufficient or insufficient $(p=.00)$. And patients more resilient turned out to be those that yes knew the test or were briefed on it. Conclusion: We can conclude that more information less anxiety and greater level of resilience against a colonoscopy.
\end{abstract}

Keywords: anxiety; resilience; colonoscopy; nursing; information; conscious sedation; deep sedation. 


\section{Introducción}

$E^{1}$ profesional sanitario no solo está presente en la realización de la colonoscopia sino que debe prestar atención los días previos, para asegurar una adecuada preparación, y los días posteriores por la posibilidad de la aparición de complicaciones. No obstante, una adecuada atención sanitaria, en concreto la atención enfermera no termina en unas destrezas técnicas, sino que se fundamenta en el cuidado humano desde un enfoque profesional y desde un punto de vista integral, situando a la persona en el centro de todo el proceso asistencial bajo una visión holística abarcando todas sus dimensiones, biológica, psicológica, social, espiritual y ecológica (Mateos, 2015).

La colonoscopia es una prueba diagnóstica y terapéutica a la vez, y constituye el estudio con mayor sensibilidad y especificidad de la patología colónica, por lo que se ha convertido en el método de elección en la mayoría de los pacientes adultos con síntomas intestinales (De la TorreArrieta, Roy-Guerrero, \& Artajona-Mata, 2014). La colonoscopia, en teoría, puede ser realizada sin sedación, y no hay reglas fijas para la premedicación. Sin embargo, esta mejora las condiciones del examen tanto para el médico como para el paciente.

En la actualidad se emplean dos modalidades de anestesia: Sedación consciente que se realiza mediante la administración de benzodiacepinas y opiáceos como el midazolan y el fentanilo (Martínez et al., 2014; Nunes Pereira das Neves et al., 2016). Y sedación profunda cuyo objetivo es aliviar el dolor y disminuir la incomodidad y los recuerdos en pacientes sometidos a un proceso doloroso. Gracias a la disponibilidad de nuevos fármacos como el propofol, se ha ido extendiendo en los últimos años la realización de la colonoscopia bajo este tipo de anestesia (Martínez et al., 2014).

El someterse a cualquier prueba diagnóstica como es la colonoscopia, lleva implícito en el paciente efectos perjudiciales o negativos a nivel emocional, desde que el paciente es citado para la prueba hasta el resultado, pasando por el desarrollo de la misma (Cardenal et al., 2008). Entre estas reacciones emocionales podemos encontrar la ansiedad, ocasionada por el miedo al dolor o el temor ante el futuro; la depresión o problemas de adaptación; o problemas de rebeldía frente a un destino no deseado (López Ibor, 2007). Los factores que influyen en esas reacciones son diversos, como la personalidad, el tipo de enfermedad, o la edad, entre otros. Es muy importante la comunicación con el paciente, donde la enfermera (a través de un lenguaje comprensible) proporciona al paciente la facilidad de participar en su recuperación (Escudero, Viña, \& Calleja, 2014).

También influye la resiliencia, definida como la capacidad de adaptarse a experiencias negativas (Rutter, 1985), que involucra procesos genéticos, biológicos, psicológicos y dinámicos (Monroy-Cortés, \& Palacios-Cruz, 2011). La resiliencia se ha llegado a considerar también como una característica esencial e inherente a la enfermera para el desarrollo de su asistencia diaria (Tusaie, \& Dyer, 2004), como consecuencia de la constante exposición a un continuo entorno laboral adverso al que debe hacer frente (Arrogante, Pérez-García, \& AparicioZaldívar, 2015), siendo los profesionales clave para enseñar dicha capacidad resiliente a los pacientes (Bermejo Higuera, 2015).

El objetivo general del presente estudio es mostrar el nivel de ansiedad y resiliencia de los pacientes que van a ser sometidos a colonoscopia, y demostrar que la información recibida sobre la prueba influye en estos niveles, además de ver la relación entre ambos factores. A nivel específico el objetivo es identificar las diferencias existentes en ansiedad y resiliencia entre hombres y mujeres; y analizar si el tipo de sedación utilizado en la colonoscopia se relaciona con el nivel de ansiedad de los pacientes.

\section{Método}

\section{Diseño}

Se diseñó un estudio observacional, descriptivo, transversal y prospectivo.

\section{Participantes}

Los participantes del estudio fueron pacientes que acudieron, para la realización de una colonoscopia, al Servicio de Digestivo y Endoscopias del Hospital Universitario de Santa Lucía y Hospital Santa María del Rosell de Cartagena (Murcia). Fueron 100 el total de los participantes, 38 hombres y 62 mujeres con edades comprendidas entre 27 y 88 años ( $M=59,45$, DT=13,75). La estrategia de muestreo fue por conveniencia, según llegaban al servicio los pacientes, en el periodo de estudio de junio a julio de 2015. A 50 se les realizó la prueba bajo sedación consciente y a 50 bajo sedación profunda. Se incluyeron a pacientes ambulatorios y hospitalizados mayores de edad que iban a ser sometidos a la realización de una colonoscopia por sintomatología (un 53\%), por antecedentes familiares (un 15\%) o por revisión (un 32\%), bajo sedación consciente o sedación profunda, y que aceptaron de manera voluntaria participar en el estudio, previa firma del consentimiento informado. Los criterios de ex- 
clusión fueron: limitaciones cognitivas que impidiesen a los pacientes entender y contestar a las preguntas del cuestionario, o que fuesen sometidos a la prueba por un proceso de urgencia.

\section{Instrumentos}

Para identificar las características o los aspectos sociodemográficos, así como el conocimiento previo acerca de la colonoscopia se elaboró un cuestionario de elaboración propia, ad-hoc, formado por 8 ítems con variables cualitativas y cuantitativas que se consideraron, tras una revisión exhaustiva de la literatura, más relevantes para el estudio. Estas variables son: tipo de sedación (consciente o profunda); sexo (hombre o mujer); edad; motivo por el que se realiza la colonoscopia (por antecedentes familiares, por sintomatología o por prevención); si se tiene conocimiento de la prueba (sí o no); si se ha recibido información sobre la colonoscopia (sí o no); quién ha informado (amigos/familiares, personal sanitario u otros); y cómo considera el nivel de la información (suficiente 0 insuficiente). Para la recogida de las variables psicológicas, se utilizaron la escala de ansiedad STAI y la escala de resiliencia RISC-10.

El Stait-Trait Anxiety Inventory (STAI) de Spielberger, Gorsuch y Lushene, fue adaptado al español por SpieIberger, Gorsuch y Lushene (1982). Este instrumento mide a través de 40 preguntas la Ansiedad Estado (AE) y la Ansiedad Rasgo (AR). Tiene un alfa de Cronbach de 0,90 para la ansiedad rasgo y un 0,94 para la ansiedad estado (Guillén-Riquelme, \& Buela Casal, 2011). La $\mathrm{AE}$ recoge los sentimientos subjetivos y transitorios de tensión, aprensión y miedo que pueden variar a lo largo del tiempo y fluctuar de intensidad. La AR refleja un estado emocional relativamente estable, refiriéndose a la tendencia general de la persona a percibir como amenazadoras situaciones cotidianas (Pifarré et al., 2011).

Para medir la resiliencia hemos utilizado la escala The Coonor-Davidson Resilience Scale CD-RISC-10 (Campbell-Sills, \& Stein, 2007), versión abreviada de ConnorDavidson Resilience Scale (CD-RISC) de Connor y Davidson. Consta de 10 ítems y el alfa de Cronbach de la versión adaptada y validada al castellano fue de 0,85, el mismo valor que se obtuvo en el instrumento original (Notario-Pacheco et al., 2011).

\section{Procedimiento}

Para la recogida de datos se les entregó a los pacientes, de forma individual, a la llegada al servicio, antes de la realización de la colonoscopia, un cuestionario de tipo autocumplimentado en presencia del investigador; que recogía las variables anteriormente mencionadas. Mientras esperaban para la realización de la prueba se les explicaba en qué consistía la investigación y si querían participar contestando a dicho cuestionario, dejándolo relleno antes de entrar a realizarse la colonoscopia. Los criterios de inclusión fueron firmar el consentimiento informado, no tener problemas con el idioma y completar la totalidad del cuestionario, quedando excluidos los casos en los que no reunían estas características.

El proyecto de este estudio cuenta con la aprobación de la Comisión de Investigación del Área 2 de Salud de la Región de Murcia. Los sujetos participantes lo hicieron de forma voluntaria y fueron informados del estudio que se iba a desarrollar, como hemos mencionado anteriormente. Leyeron y firmaron una carta de consentimiento con la que verificaban su voluntariedad de participar en el estudio. De esta manera, se codificó cada cuestionario con el fin de preservar el anonimato y confidencialidad de los datos recogidos según la declaración de Helsinki.

\section{Análisis estadístico}

Para procesar la información se elaboró una base de datos con el programa informático SPSSC v21. Para el análisis descriptivo de la variables sociodemográficas así como del conocimiento previo acerca de la colonoscopia se utilizaron estimadores de tendencia central (media y desviación típica) y proporciones y porcentajes, según el tipo de variable. Para la comparación de variables cuantitativas entre grupos se empleó la T de Student, y la Chi-Cuadrado de Pearson para la comparación de las variables cualitativas. Antes de realizar las pruebas se comprobó el supuesto de normalidad de las variables. Para verificar que se cumple esta condición, se aplicó a todas ellas el test de Kolmogorov-Smirnov. Además, se calculó el tamaño del efecto a través de la d de Cohen para valorar la magnitud del efecto de la intervención. Para el análisis de la relación entre las escalas de la ansiedad y de resiliencia se hizo mediante el análisis de correlación de Spearman siendo construida una única matriz. A efectos de interpretación y análisis de los datos el nivel de confianza asumido fue de $0,05(p<0,05)$.

\section{Resultados}

El $18 \%$ de los pacientes tenían AR elevada, sin embargo, el $52 \%$ presentaban AE elevada ante la realización de la colonoscopia. El $21 \%$ obtuvo una puntuación baja en resiliencia, frente al $79 \%$ que mostró una adecuada resiliencia. El $89 \%$ de los pacientes refieren que sí saben en qué consiste la colonoscopia, frente al $11 \%$ que no. El $69 \%$ señala que sí ha recibido información, frente al 31\% que 
no. Un $54 \%$ ha recibido información de algún profesional sanitario, un $18 \%$ la ha recibido de familiares o amigos y el resto, un $28 \%$, no ha recibido información de nadie. Un $57 \%$ considera insuficiente la información recibida frente a un $43 \%$ que la considera suficiente.

Tras realizar la correlación entre el nivel de resiliencia y la $A E$ se obtuvo una significación estadística negativa $(\rho=-.309, p=0,00)$ al igual que entre la resiliencia y la AR $(\rho=-.305, p=0,00)$.

\section{Ansiedad Estado, Ansiedad Rasgo Resiliencia en hombres y mujeres}

En relación a la variable sexo, en la tabla 1 podemos observar la media obtenida por hombres y mujeres en la $A E$ y la AR.

Tabla 1. Puntuación media total en AE y AR en hombres y mujeres.

\begin{tabular}{|l|c|c|c|c|}
\hline & Sexo & N & M & DT \\
\hline Ansiedad & Hombre & 38 & 26,55 & 9,63 \\
Estado & Mujer & 62 & 29,74 & 11,64 \\
\hline Ansiedad & Hombre & 38 & 21,92 & 7,22 \\
Rasgo & Mujer & 62 & 24,31 & 9,38 \\
\hline
\end{tabular}

Aunque podemos apreciar medias superiores en $A E$ y AR en las mujeres, no se obtuvo una relación estadísticamente significativa, ni en $\operatorname{AE}(p=0,16)$ ni en $\operatorname{AR}(p=0,18)$. Tampoco obtuvimos una relación estadísticamente significativa entre resiliencia y sexo $(p=0,62)$, donde los hombres obtuvieron un porcentaje mayor que las mujeres en resiliencia, un $81,6 \%$ frente a un $77,4 \%$.

\section{Ansiedad Estado, Ansiedad Rasgo y Resiliencia según el conocimiento y la información recibida de la colonoscopia}

La puntuación media total obtenida en la $\mathrm{AE}$ en relación a los pacientes que señalaron que sí conocían la prueba fue de 27,71 (DT=11,19) frente a los que contestaron que no, que presentaron una puntuación media mayor en $\mathrm{AE}$ $(M=35,18, D T=0,19)$. Se comprobó que existía una relación significativa a nivel estadístico entre la ansiedad y el conocimiento o no de la prueba en el momento de la colonoscopia $(F=6,93, p=0,01)$.

Con respecto a la $A R$, los pacientes que sí conocían la prueba obtuvieron una puntuación media relacionada con ansiedad normal de 23,08 , frente a los que señalaron que no la conocian, que obtuvieron una puntuación media de 26 , correspondiente también a un nivel de ansiedad nor- mal. En este caso no se obtuvo una relación estadísticamente significativa $(F=2,97, p=0,08)$.

En la tabla 2 observamos la puntuación media total obtenida en AE y AR en relación a los pacientes que señalaron que sí obtuvieron información sobre la prueba y los que no, los cuales muestran una media de $A E$ y AR mayor.

Tabla 2. Puntuación media total en $A E$ y $A R$ en los pacientes que recibieron información sobre la colonoscopia y los que no.

\begin{tabular}{|l|c|c|c|c|}
\hline & $\begin{array}{c}\text { Recibió } \\
\text { información }\end{array}$ & N & M & DT \\
\hline Ansiedad & $\mathrm{Si}$ & 69 & 25,64 & 10,30 \\
Estado & No & 31 & 34,97 & 9,77 \\
\hline Ansiedad & $\mathrm{Si}$ & 69 & 22,01 & 8,86 \\
Rasgo & No & 31 & 26,48 & 7,42 \\
\hline
\end{tabular}

Se obtuvo significación estadística entre la $A E$ y el haber recibido o no información sobre la prueba $(F=1,70$, $p=0,00)$, no ocurrió lo mismo con la $A R(F=1,41, p=0,16)$.

De la misma manera, se obtuvo la puntuación media total en AE y AR de la percepción que tenían los pacientes en cuanto al nivel de información recibida. Podemos ver en la tabla 3 que los que consideran haber recibido un nivel insuficiente de información presentan medias superiores a los que consideran haber tenido suficiente información, tanto en AE como en AR.

Tabla 3. Puntuación media total en AE y AR en los pacientes que consideraron el nivel de información recibida como suficiente e insuficiente.

\begin{tabular}{|l|c|c|c|c|}
\hline & $\begin{array}{c}\text { Nivel de } \\
\text { información } \\
\text { recibida }\end{array}$ & N & M & DT \\
\hline Ansiedad & Suficiente & 43 & 22,88 & 9,64 \\
Estado & Insuficiente & 57 & 32,79 & 10,03 \\
\hline Ansiedad & Suficiente & 43 & 21,07 & 9,31 \\
Rasgo & Insuficiente & 57 & 25,16 & 7,77 \\
\hline
\end{tabular}

Se obtuvo una relación estadísticamente significativa entre la ansiedad, $A E(F=0,11, p=0,00), A R(F=0,88$, $p=0,01$ ), y el nivel de información recibida.

Respecto al nivel de resiliencia, aunque no se obtuvo una relación significativa a nivel estadístico $(p=0,12)$, los que señalaron que sí conocían en qué consistía la prueba obtuvieron una puntuación media superior $(M=26,13$, $\mathrm{DT}=8,10)$ en el nivel de resiliencia que los que contestaron que no ( $M=23,27, D T=5,33)$. 
Los pacientes que señalaron que no obtuvieron información sobre la prueba mostraron una media de resiliencia menor $(M=23,68, D T=7,43)$ que los que señalaron que sí la obtuvieron ( $M=26,78, D T=7,93)$. Aunque no se obtuvo una relación estadísticamente significativa $(p=0,58)$.

Los que consideran haber recibido un nivel suficiente de información, presentan medias superiores en la resiliencia $(M=27,19, D T=8,53)$ que los que consideran haber recibido insuficiente información $(M=24,79$, $D T=7,24)$. Aunque no se obtuvo una relación estadísticamente significativa $(p=0,20)$.

\section{Nivel de ansiedad y tipo de sedación}

Los pacientes que se iban a realizar la colonoscopia bajo sedación "profunda", obtuvieron una puntuación media total en $A E(M=30,18, D T=11,74)$ y $A R(M=24,74, D T=8,60)$ mayor que los que se iban a realizar la prueba con sedación "consciente", con una AE (M=26,88, DT=10,01) y AR $(M=22,06, D T=8,59)$ menor. Sin embargo, no se obtuvo una relación estadísticamente significativa ni en la $A E$ $(p=0,13)$ ni en la AR $(p=0,12)$.

\section{Discusión y conclusiones}

La realización de una colonoscopia, a pesar de sus beneficios, no deja de ser un procedimiento incómodo para el paciente, donde el dolor, el miedo a lo desconocido, así como la sensación por parte del paciente de "violación a su intimidad", han hecho que este procedimiento genere cierto grado de ansiedad, convergiendo frecuentemente en el rechazo, bien por una mala experiencia anterior o referencias al respecto (Chatman, Sutherland, Van der Zwan, \& Abraham, 2013; Hernández-Calzada, Cordero-Escobar, RiveroMoreno, Fernández Navarro, \& Mora-Díaz, 2015). En nuestro estudio la mitad de los pacientes que iban a someterse a una colonoscopia presentaron ansiedad estado (un 52\%).

Varios estudios, que utilizaron el cuestionario STAI, hallaron cifras elevadas de ansiedad en pacientes sometidos a pruebas diagnósticas (Doménech et al., 2010; Pifarré et al., 2011). Cabello-Fernández et al. (2014) obtuvieron más puntuación en la $\mathrm{AE}$ en los hombres que en las mujeres, ante una colonoscopia. También se obtuvieron resultados similares en otro estudio (Pifarré et al., 2011), sin embargo, en nuestro caso, se hallaron medias superiores en AE y AR en las mujeres, aunque no se obtuvo relación estadísticamente significativa. En el caso de la resiliencia nos encontramos un porcentaje mayor de la misma en hombres, aunque tampoco hallamos diferencias estadísticamente significativas.

Herranz-García (2015) concluyó que el 63,07\% de los pacientes que acudían al servicio de medicina nuclear por primera vez presentaban ansiedad en el momento de la prueba. De éstos, el 52,30\% de los pacientes reconocían que la información recibida había sido escasa. En nuestro caso el $57 \%$ consideraron insuficiente la información recibida. La falta de información es un motivo de ansiedad en los pacientes que se van a realizar una prueba diagnóstica. Rollbusch, Mikocka-Walus y Andrews (2014) comprobaron que algunos pacientes no asimilaron la información correcta sobre la colonoscopia, por lo que, concluyeron lo importante que resulta, por parte del personal sanitario, cerciorarse de dicho altercado. Voiosu et al. (2014) obtuvieron, tras evaluar a 452 pacientes, que el $70,2 \%$ consideraban la información proporcionada antes de la colonoscopia como insuficiente, concluyendo que la satisfacción y la preocupación del paciente va influenciada con el nivel de información y con la relación médico-paciente.

Al ser la colonoscopia una técnica invasiva, sujeta a posibles complicaciones (Chan et al., 2015), necesita de un consentimiento informado y es a través de este donde se detalla la información sobre la prueba. Un estudio concluyó que una combinación de información verbal y escrita antes de procedimientos endoscópicos consigue menores niveles de ansiedad, ya que mejora el nivel de entendimiento de la prueba en sí y de sus posibles complicaciones (Sanguinetti et al., 2015). Otro estudio resalta que el formato escrito es obligatorio por ley, pero el más adecuado sería el oral, ya que refuerza la relación entre el paciente y el médico (Heras-Meseguer, 2015).

A pesar de ser la información al paciente un tema bastante estudiado, aún se conoce la carencia existente de la misma. En nuestro estudio, se muestra que el nivel de ansiedad, de forma significativa, se relaciona con la información y el conocimiento sobre la colonoscopia, así como con un mejor nivel de resiliencia en aquellos pacientes que poseían una información y un conocimiento suficiente sobre la prueba a la que se iban a someter. Por lo tanto, señalamos la importancia de ofrecer una información suficiente, clara y adecuada para que los pacientes puedan poder expresar opiniones y hacer preguntas. Ya sea mediante el consentimiento informado (parte ética y práctica importante de la atención informada) o reforzada con folletos escritos, videos, etc. (Kinnersley et al., 2013). Por lo tanto, a partir de los resultados obtenidos, proponemos nuevas líneas de investigación para identificar información que demanda el paciente con el fin de contribuir en el avance de la disciplina enfermera y mejorar la calidad asistencial en todos los niveles. 
Hay estudios que demuestran también la necesidad de informar al paciente sobre la anestesia a la que va a ser sometido (Ortiz, Wang, Elayda, \& Tolpin, 2015). En nuestro estudio, ante la sedación profunda se mostraron niveles de $A E$ más elevados que ante la sedación consiente, esto puede deberse tal vez a la reiterada mención de la falta de conocimiento, en este caso, sobre la anestesia o el tipo de sedación a la que se iban a someter, aumentando de esta forma sus miedos; de cualquier forma, faltaría investigar otras posibles variables influyentes en dicha ansiedad. Pues en otros estudios se ha mostrado el paciente más satisfecho con la sedación profunda que con la sedación consciente (De la Torre-Arrieta et al., 2014; Nunes Pereira das Neves et al., 2016), por considerarse como protector en las molestias percibidas por el paciente.

No se ha encontrado ningún estudio que evalúe el nivel de resiliencia en pacientes que se van a realizar una colonoscopia o cualquier otra prueba diagnóstica, así como la relación que pueda existir con el conocimiento de la prueba o la información recibida. En nuestro estudio aparece una correlación negativa de la $A E$ con la resiliencia, es decir, si existe poca resiliencia resultará más difícil controlar los niveles de ansiedad u otros problemas (Saavedra, Castro, \& Inostroza, 2012).

Como limitaciones al estudio cabría mencionar el contar con un grupo control, en donde hubiésemos medido el nivel de ansiedad y resiliencia en pacientes que acudiesen al servicio de digestivo por otros motivos distintos a la realización de la colonoscopia.

Podemos concluir, a partir de nuestros resultados ante los participantes en este estudio, que existe una relación entre dar información sobre la prueba y mostrar menos niveles de ansiedad y más resiliencia. Que se dio una correlación negativa entre la resiliencia y la ansiedad. Y que en relación al tipo de sedación utilizado ante la colonoscopia y su influencia en la ansiedad del paciente no obtuvimos significación estadística, al igual que tampoco hallamos diferencias significativas a nivel estadístico entre hombres y mujeres, ni en los niveles de ansiedad ni en los de resiliencia.

\section{Referencias Bibliográfica}

Arrogante, O., Pérez-García, A.M., \& Aparicio-Zaldívar, E.G. (2015). Bienestar psicológico en enfermería: relaciones con resiliencia y afrontamiento. Enfermería Clínica, 25(2), 73-80.

Bermejo Higuera, J.C. (2015). Resiliencia y cuidado enfermero. Revista ROL de Enfermería, 38(6), 408-413.

Cabello-Fernández, A., Franch-Almela, P., CabanesHernández, E., Arnau-Paradís, T., Cabañas-Carrasco, M.P., \& Martínez-Vinuesa, L. (2014). Aplicación de la encuesta de ansiedad STAI (state-trait anxiety inventory) para valorar la necesidad de sedación en pacientes de cribado de cáncer colorrectal. Enfermería en Endoscopia Digestiva, 1(2), 3-5.

Campbell-Sills, L., \& Stein, M.B. (2007). Psychometric analysis and refinement of the Connor-Davidson Resilience Scale (CD-RISC): validation of a 10-item measure of resilience. Journal of Traumatic Stress, 20(6), 1019.

Cardenal, M.C., Rodríguez, A., Cruzado, J.A., González, A., González, M.J., \& Roa, A. (2008). Ansiedad, depresión y calidad de vida durante las fases de cribado mamográfico en mujeres de la comunidad de Madrid. Psicooncología, 5(1), 129-146.

Chan, A.O., Lee, L.N., Chan, A.C., Ho, W.N., Chan, Q.W., Lau, S., \& Chan, J.W. (2015). Predictive factors for colonoscopy complications. Hong Kong Medical Journal, 21, 23-9.

Chatman, N., Sutherland, J.R., Van der Zwan, R., \& Abraham, N. (2013). A survey of patient understanding and expectations of sedation/anaesthesia for colonoscopy. Anaesth Intensive Care, 41(3), 369-73.

De la Torre-Arrieta, I., Roy-Guerrero, L., \& ArtajonaMata, R.M. (2014). Calidad en colonoscopia: grado de satisfacción del paciente. NURE Investigación, 69, 2-15.

Doménech, A., Notta, P., Benítez, A., Ramal, D., RodríguezBel, L., Massuet, C., \& Martín-Comín, J. (2010). Evaluation of the anxiety state in patients receiving radioiodine treatment or who undergo a sentinel lymph node examination in the Nuclear Medicine Department. Revista Española de Medicina Nuclear, 29(2), 63-72.

Escudero, D., Viña, L., \& Calleja, C. (2014). For an opendoor, more comfortable and humane intensive care unit. It is time for change. Medicina Intensiva, 38(6), 371-375.

Fernández-Lansac, V., Crespo-López, M., Cáceres, R., \& Rodríguez-Poyo, M. (2012). Resiliencia en cuidadores 
de personas con demencia: estudio preliminar. Revista Española de Geriatría y Gerontología, 47(3), 102-109.

Guillén-Riquelme, A., \& Buela Casal, G. (2011). Actualización psicométrica y funcionamiento diferencial de los items en el State Trait Anxiety Inventory (STAI). Psicothema, 23(3), 510-515.

Heras-Meseguer, M. (2015). Influencia del protocolo de información en la ansiedad estado en pacientes sometidos a la extracción de terceros molares inferiores incluidos. Tesis doctoral inédita. Universidad de Sevilla.

Hernández-Calzada, J.J., Cordero-Escobar, I., RiveroMoreno, M., Fernández-Navarro, M.A., \& Mora-Díaz, I. (2015). Sedación consciente para la colonoscopia ambulatoria. Revista Cubana de Anestesiología y Reanimación, 14(1), 13-28.

Herranz-García, N. (2015). Valoración del estado de ansiedad de los pacientes que van a medicina nuclear por primera vez. Tesis doctoral inédita. Universidad de Jaén.

Kinnersley, P., Phillips, K., Savage, K., Kelly, M.J., Farrell, E., Morgan, B.,..., Edwards, A.G. (2013). Interventions to promote informed consent for patients undergoing surgical and other invasive healthcare procedures (Review). The Cochrane Database Systematic Reviews, 7, 1-252.

López Ibor, M.I. (2007). Ansiedad y depresión, reacciones emocionales frente a la enfermedad. Anales de Medicina Interna, 24(5), 209-211

Martínez, O., Ballesteros, D., Estébanez, B., Chana, M., López, B., Martín, C... Blancas, R. (2014). Características de la sedación profunda en procesos endoscópicos gastrointestinales, realizadas por médicos intensivistas. Medicina Intensiva, 38(9), 533-540.

Mateos, A. (2015). Abordaje psicológico del paciente con enfermedad inflamatoria intestinal. Tesis doctoral inédita. Universidad de Valladolid.

Monroy-Cortés, B.G., \& Palacios-Cruz, L. (2011). Resiliencia: ¿Es posible medirla e influir en ella? Salud Mental, 34, 237-246.

Notario-Pacheco, B., Solera-Martínez, M., SerranoParra, M.D., Bartolomé-Gutiérrez, R., Garcia-Campayo,
J., \& Martinez-Vizcaino, V. (2011). Reliability and validity of the Spanish versión of the 10 item Connor-Davidson Resilience Scale (10-item CD-RISC) in young adults. Health Qual Life Outcomes, 9-63.

Neves, J.F.N.P., Araújo, M.M.P.N., Araújo, F.P., Ferreira, C.M., Duarte, F.B.N., Pace, F.H., ...Ferreira, L.E.V.V.C. (2016). Sedação para colonoscopia: ensaio clínico comparando propofol e fentanil associado ou njo ao midazolam. Revista Brasileira de Anestesiologia, 66(3), 231-236.

Ortiz, J., Wang, S., Elayda, MA., \& Tolpin, DA. (2015). Información preoperatoria al paciente: ¿podemos mejorar la satisfacción y reducir la ansiedad? Revista Brasileira de Anestesiologia, 65(1), 7-13.

Pifarré, P., Simó, M., Gispert, J.D., Pallarés, M.D., Plaza, P., \& Martínez-Miralles, E. (2011). Diagnostic Imaging Studies: Do They Create Anxiety? Revista Española de Medicina Nuclear, 30(6), 346-350.

Rollbusch, N., Mikocka-Walus, AA., \& Andrews, J.M. (2014). The Experience of Anxiety in Colonoscopy Outpatients:A mixted-MethodAStudy. Gastroenterology Nursing, 37(2), 166-175.

Rutter, M. (1985) Resilience in the face of adversity: protective factors and resistance to psychiatric disorder. British Journal Psychiatry, 147, 598-611.

Saavedra, E., Castro, A., \& Inostroza, A. (2012). Niveles de resiliencia en adultos diagnosticados con y sin depresión. Revista Pequén, 2(1), 161-184.

Sanguinetti, J.M., Lotero-Polesel, J.C., Iriarte, S.M., Ledesma, S.M., Canseco-Fuentes, S.E., \& Caro, L.E. (2015). Informed consent in colonoscopy: A comparative analysis of 2 modes. Revista de Gastroenterología de México, 80(2), 144-149.

Serrano-Parra, M.D., Garrido-Abejar, M., Notario-Pacheco, B., Bartolomé-Gutiérrez, R., Solera-Martínez, M., \& MartínezVizcaíno, V. (2013). Validez de la escala de resiliencia Connor-Davidson (10 items) en una población de mayores no institucionalizados. Enfermería Clínica, 23, 14-21.

Spielberger, C.D., Gorsuch, R.L., \& Lushene, R. (1982). Manual del Cuestionario de Ansiedad Estado/ Rasgo (STAl). Madrid: TEA Ediciones.

Tusaie, K., \& Dyer, J. (2004). Resilience: a historical 
review of the construct. Holistic Nursing Practice, 18, 3-8.

Voiosu, A., Tantau, A., Garbulet, C., Tantáu, M., Mateescu, B., Báicus, C.,... Vojosu, T. (2014). Factors affecting colonoscopy comfort and compliance: a questionnaire based multicenter study. Romaniam Journal of Internal Medicine, 52(3), 151-7. 